\title{
Biomedical Response and Different Applications of Magnetic Nanomaterials
}

\author{
Bhunia $\mathrm{AK}^{1 *}$ and Saha $\mathrm{S}^{2}$ \\ ${ }^{1}$ Department of Physics, Government General Degree college at Gopiballavpur-II, India \\ ${ }^{2}$ Department of Physics and Technophysics, Vidyasagar University, India
}

Submission: June 05, 2018; Published: August 30, 2018

*Corresponding author: Bhunia AK, Department of Physics, Government General Degree College, Gopiballavpur-II, Beliaberah-721517, India, Email: amitphysics87@gmail.com

\begin{abstract}
From the ultimate decade, nanoscience and nanotechnology have been multiple explored for wide studies in medical science. The bodily principles vital a few contemporary biomedical programs of magnetic nanoparticles (MNPs) are inspected. Starting from basic magnetic concepts of MNPs which are responsible for biomedicine, and drawing on examples from biomedicine, the relevant physics of MNPs and their responses to implemented magnetic fields are inspected. This mini review discusses about the different responsible properties for biomedicine and the various biomedical applications of MNPs as reported by the different researchers.
\end{abstract}

Keywords: Magnetic fields; Biomedical programs; Protein; Nanoparticles; Hyperthermia treatments

\section{Mini Review}

Magnetic nanoparticles (MNPs) are attractive for biomedicine because of their natural influence by magnetic field and different optical, magnetic properties [1-3]. In the nanoscale their dimensions are comparable and smaller to those of a cell $(10-100 \mu \mathrm{m})$, a virus $(20-450 \mathrm{~nm})$, a protein $(5-50 \mathrm{~nm})$ or a gene ( $2 \mathrm{~nm}$ wide and $10-100 \mathrm{~nm}$ long). The common MNPs are nickel, cobalt, iron and their chemical compounds. Magnetic nanoparticles had been researched drastically seeing that they have got appealing traits that might be used in nano based catalysis. They used for different applications: Magnetic resonance imaging, Biomedicine Magnetic particle imaging, Data storage Nanofluids, Environmental remediation, Optical filters [45]. They are suitable for multi-compound detection by magnetic relaxation. Magnetic nanoparticles are useful for functional MRI studies [6]. The nanoparticles are magnetic, because of this that they obey Coulomb's law, and may be employed by way of an outside magnetic field gradient. The magnetic nanoparticles can be made to resonantly respond to a time-varying magnetic field, with advantageous consequences associated with the transfer of electricity from the exciting field to the nanoparticle. Different programs are made to be had in biomedicine as a result of the unique bodily homes of magnetic nanoparticles. on this review we will cope with the underlying physics of the biomedical applications of magnetic nanoparticles. we are able to don't forget few packages: magnetic separation, drug delivery, hyperthermia treatments and magnetic resonance imaging (MRI) contrast enhancement.

\section{Basic Concepts}

The magnetic behaviour of a blood vessel into which some magnetic nanoparticles have been injected can be understood from the fundamental concepts of magnetism. When a magnetic material/nanomaterial is placed in a field $\mathrm{H}$, the magnetic induction is $B=\mu_{0}(H+M)$. Magnetic materials are conveniently classified in terms of their volumetric magnetic susceptibility, $\chi$, where, $\mathrm{M}=\chi \mathrm{H}$. The magnetism is different for different class of magnetic materials. In paramagnetic nano materials $\chi$ varies in the range $10^{-6}-10^{-1}$. In the diamagnets $\chi$ is the range $10^{-6}$ to $-10^{-3}$. Ferromagnets, ferrimagnets and antiferro-magnetic materials exhibit ordered magnetic states and are magnetic even without a field applied. right here the prefix refers back to the nature of the coupling interplay among the electrons in the material. This coupling can deliver upward thrust to large spontaneous magnetizations; in ferromagnets $\mathrm{M}$ is typically $10^{4}$ instances larger than would seem otherwise. The susceptibility in ordered substances relies upon on temperature $\mathrm{T}$ and also $\mathrm{H}$, which offers upward push to the characteristic hysteresis of the M-H/B-H curve, with $\mathrm{M}$ approaching a saturation value at large values of $\mathrm{H}$.

The irreversibility in the hysteresis process is related to the pinning of magnetic domain walls at impurities or grain boundaries within the material, magnetic anisotropy of the crystalline lattice. The form of the hysteresis loops is decided in part with the aid of particle size [7]. For huge particles (of the order micron size or more) there's a multi-area ground country which leads to a slim hysteresis loop since it takes relatively little 
area power to make the area walls circulate. Smaller particle gives rise single domain ground state which leads to a broad hysteresis loop. For few nanometre length (of the order of tens of nanometres or less) you can see superb para-magnetism, wherein the magnetic moment of the particle as an entire is unfastened to vary in response to thermal electricity, at the same time as the individual atomic moments maintain their ordered nation relative to every other. This leads to the anhysteretic, however nevertheless sigmoidal, M-H curve. The injected nanonmaterial's perform a single domain/multi domain or super paramagnetic (SPM) B-H (or $\mathrm{M}-\mathrm{H}$ ) curve. This performance depends upon the size of the injected magnetic nanoparticles. The magnetic response of the blood Vessel itself be composed of both paramagnetic (PM) and diamagnetic (DM) response.

The PM response arises from the iron-containing haemoglobin molecules. The DM response arises from those intra-vessel proteins that include best carbon, hydrogen, nitrogen and oxygen atoms. It must be noted that the magnetic signal from the injected nano-particles (any size), a long way exceeds that from the blood vessel itself. This progressed selectivity is one of the superb features of biomedical applications of magnetic nanoparticles. The hysteresis curve of the ferromagnetism showed that energy is wanted to conquer the barrier to domain wall motion imposed via the intrinsic anisotropy, atomic impurities and grain boundaries in the nanostructure material. This energy is added by using the applied field, proportional with the $\mathrm{M}-\mathrm{H}$ (or B-H) curve. Therefore, if we apply varying (time) magnetic field to the ferromagnetic nanoparticles, we found a scenario in which there may be a regular go with the flow of power into that nanoparticles, which will defencelessly be transferred into thermal energy. This is the basic principle of hyperthermia treatments. Only magnetic field gradient exerts a pressure at a distance. Uniform field produce torque, however no translational movement. The magnetic force on a magnetic dipole $(\mathrm{m})$ :

$$
\mathrm{F}_{\mathrm{m}}=(\mathrm{m} . \boldsymbol{\nabla}) \mathrm{B}
$$

Hence, $F$ depends on the direction of $m$. For $m=\left(0,0, m_{z}\right), m$. $\boldsymbol{\nabla}=\mathrm{m}_{\mathrm{z}}(\delta / \delta \mathrm{z})$. A force will be skilled on the dipole provided there may be a field gradient in B inside the z- route. within the case of a magnetic nanoparticles dangled in a weakly DM medium (like water), the total moment on the nanoparticle is $m=V_{m} M$, where $V_{m}$ = volume of the particle and $M=$ volumetric magnetization $=\Delta \lambda H$, where $\Delta \lambda=\lambda_{\mathrm{m}}-\lambda_{\mathrm{w}}=$ effective susceptibility of the particle relative to DM medium (water). For the case of a dilute suspension of nanoparticles in natural water, we are able to approximate the general response of the particles plus water machine through $\mathrm{B}=\mu_{0} \mathrm{H}$, in order that equation (1) will become

$$
\begin{aligned}
& F_{m}=V_{m} \Delta \chi \nabla\left(\frac{B^{2}}{2 \mu_{0}}\right) o r \\
& F_{m}=V_{m} \Delta \chi \nabla\left(\frac{1}{2} B . H\right),
\end{aligned}
$$

Here we use the formula, Maxwell equation $\boldsymbol{\nabla} \times \mathrm{B}=0$, magnetostatic field energy density $=1 / 2 B . H$, and $\boldsymbol{\nabla}(B . B)=2(B . \nabla)$ $B$ as $\boldsymbol{\nabla}(A \cdot B)=(A . \nabla) B+(B . \nabla) A+A \times(\nabla \times B)+B \times(\nabla \times A)$.
Hence, Sign of $\Delta \lambda$ represent the direction of force of the energy density scalar field. $\Delta \lambda$ may be positive or negative depends on the magnetism. This explains why some materials attracted towards the pole of a permanent bar magnet. it is also the premise for the biomedical programs of magnetic separation and drug shipping (Figure 1).

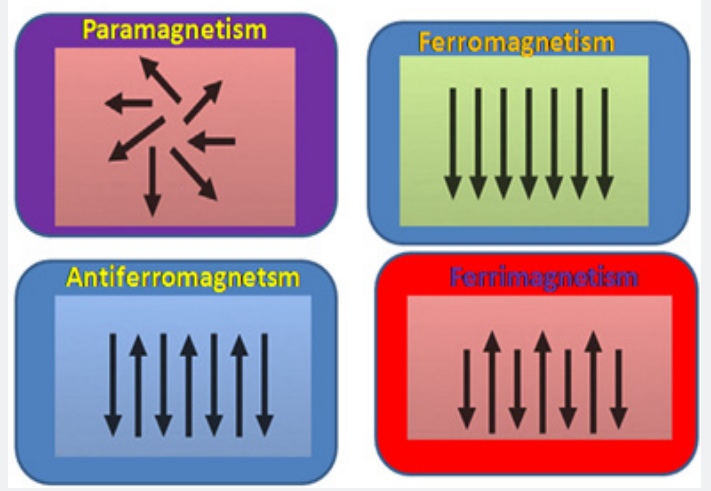

Figure 1: Alignment of the magnetic moment different magnetic structures.

\section{Biomedical Applications of Magnetic Nanoparticles}

Currently, magnetic oxides nanoparticles are taken into consideration within the field of scientific diagnostic and therapeutic strategies, which includes drug transport, magnetic resonance imaging (MRI) contrast enhancement and hyperthermia treatments [8-15]. Magnetic oxide nanoparticles are extensively utilized in drug delivery and MRI contrast agents. The magnetic contrast agents are using to become aware of the difference among the ordinary and bizarre tissues $[16,17]$. The different applications are discussed in bellow. Magnetic nanoparticles are used in biomedicine separation method. Magnetic separation based on MNPs is cheap and efficient than centrifugation or filtration [18]. The conjugation of the MNPs and target biomolecule can be without problems attracted by way of the applied magnetics area. Hence, the specified biomolecules can be easily extracted from the pristine mixture. NAs are effortlessly remoted and recovered with the aid of elution procedure. Functionalized MNPs are using for one of a kind target. This technique has been already applied for separation and purification of protein, virus, cells, bacteria's. Cano et al. [19] Showed the iron oxide nano particle-based elimination of fatty acids from organic answers and vegetable oils Banis et al. [20]. Discuss about the progressive application of super-paramagnetic iron oxide nanoparticles for magnetic separation. MNPs are used in Magneti resistive sensors for biosensing application. Magnetic sensors technique is superior than calorimetric or electrochemical technique for biomarker detection and quantifications [21]. The sensitivity and long-time stability are the key of such sensor. As a result, the method has wide inference in clinical diagnosis, food industry and environmental control. The magnetic nanoparticles are bind to the sensor surface by the mode of direct labelling and indirect labelling [21].

Therapeutic compounds with high cytotoxic activities are used in the cancer chemotherapeutic treatment. They mainly kill 
tumor cells. MNPs/therapeutic agent co-complicated are injected into the blood-flow and guided to consciousness over the tumor region through external applied inhomogeneous magnetic fields [14]. The therapeutic agents packed in the NMPs are then released to wreck the tumor cellular successfully. targeted transport of drug molecules with MNPs can enhance the biodistribution and guard the drugs from the microenvironment. Molecules showing higher internalization through most cancers cells than wholesome cells and allowing the usage of the therapeutic marketers at low sufficient doses to reduce the toxicity of chemotherapy. some of studies have tested the advantages of the usage of MNPs for drug shipping [22].

MRI is the most effective diagnostic techniques in medical fields MRI is a non-invasive technique that makes use of magnetic fields to produce high decision and excessive-assessment images of tissue structure and characteristic. MNPs are used for MRI contrast agents [23]. In MRI the density of each tissue and blood flow in vessels can be noted. MRI is superior than CT, PET or radiological imaging. Our body contain nearly $70 \%$ water inside tissues. The density of the contain water inside tissues are different for different tissues. The unpaired spins of hydrogen atom (1s1) in the water molecules are differently reacted to the applied subject in each tissue. The MR pix are formed based totally at the returning time evaluation of different tissues. In MRI, applied RF frequency induces the relaxation of proton spins. There are two relaxations processes, longitudinal relaxation (T1-recovery) and transverse relaxation (T2-decay).

Generally, $\mathrm{Gd}^{3+}$ or $\mathrm{Mn}^{2+}$ complexes are used for $\mathrm{T} 1$ contrast agents. These contrast agent display toxicity due to the presence of steel ions and raise new environmental issues. On the other aspect, MNPs with advanced efficiency, dispensability, and biocompatibility, belonging to the $\mathrm{T} 2$ comparison sellers, have thus been the problem of severa investigations as capacity MRI contrast retailers. Iron oxide nanoparticles are used as assessment agents in MRI [24]. Manuel Pernia Leal showed that water soluble MNPs display a high dense Polyethylene Glycol (PEG) center shell and permit long blood flow instances up to $24 \mathrm{~h}$. The mixture of low toxicity, remarkable T2 rest times, collectively with the lengthy move instances, makes those magnetic nanomaterials very promising evaluation marketers for MRI-primarily based molecular imaging in medical exercise. Gold-covered iron nanoparticles are used as magnetic resonance agent for $\mathrm{T} 1$ and T2 weighted imaging [25].

Recently SPIONs are the most-appropriate candidates for MRI contrast agents [26]. Suitable coating layer on the particle surface is needed to improve the biocompatibility. The utilizable coating layers can be of various kinds such as polymers like dextran, metals like gold, and ceramics like $\mathrm{SiO}_{2}$. Many times, dextran, citric acid, and carbohydrate chains are used for coating on SPIONs surface [27]. Recently SPION stabilized by alginate (SPION-alginate) used as a contrast agent to improve the sensitivity of MRI [28].

Magnetic nanoparticles and their composites with different multifunctional materials are used for drug delivery agents [29]. In the targeted drug delivery process, the movement and distribution of drug in frame are managed and pushed to big places that incorporate tumors [30]. On this method, the collections of drug/ magnetic vendors (magnetic nanoparticles) are injected to blood flow, after which via applying the external magnetic discipline that produces magnetic gradient in special spots of body (tumors), the drug/carrier sets are transferred and proven in tumors [31,32]. For such applications size of the nanoparticles should small and within the superparamagnetic behavior range such that they can move more freely in vessels and they are detected by immune system rarely; thus, their retention time in blood is augmented. For such uses the minimum Hc and $M_{\mathrm{r}}$ with high of $M \mathrm{~s}$ are better for targeting. Biocompatibility of magnetic nano carriers is greatly important for this in vivo application. According to reported resuls, $\mathrm{Fe}_{3} \mathrm{O}_{4}$ is an ideal fitting candidate for magnetic Drug Delivery. $\mathrm{Lv}$ et al. used $\gamma-\mathrm{Fe}_{2} \mathrm{O}_{3} / \mathrm{SiO}_{2}$-capsule composites for drug delivery and magnetic targeting system [33]. Caetano et al. studied the magnetic hyperthermia-induced drug release from ureasil-PEO$\gamma-\mathrm{Fe}_{2} \mathrm{O}_{3}$ nano composites [34]. Quan et al. [35] Used HSA coated iron oxide nanoparticles as drug delivery vehicles for cancer therapy [35]. Chetok et al. [36] Showed that Iron oxide nanoparticles worked as a drug delivery vehicle for MRI monitored magnetic targeting of brain tumors.

Hyperthermia is a medical treatment that selectively kill the tumor cells over healthful cells via heating a tumor at temperatures among $42^{\circ} \mathrm{C}-46^{\circ} \mathrm{C}$. it can be complete frame hyperthermia or localized hyperthermia [37] in the MNPs-based hyperthermia treatment, ferromagnetic or SPM particles are operating to generate warmness into the tumor tissue, observed by way of irradiation the use of an Alternating cutting-edge (AC) magnetic area. MNPs are increasing the fulfillment of hyperthermia. MNPs used for hyperthermia are in the variety of few tens of nanometer in length, accordingly allowing easy attain into tumors after intravenous injection with the aid of the carried out magnetic area. The MNPs customize with most cancers-precise binding marketers permit them being cantered in the direction of specific tumor tissues. Li el al. used low frequency magnetic field for studied the magnetic nanoparticle-based evaluation of tumor treatment [38].

\section{Conclusion}

The blessings of MNPs permit for its capability application in biomedicine. The concept of using MNPs at the biomedicine programs substantially blessings from the rapid improvement of the nanoscience and nanotechnology. Biocompatibility is a venture for such biomedical applications. Their focused-on potential and biocompatibility can be advanced thru floor coating. The surface coating needs to be selected with extreme care to suit the favoured floor characteristics for a selected biomedical utility research at the capability biomedical packages is just on the start of the street with tremendous challenges. For higher biomedical, further studies continues to be needed to apprehend the physicochemical properties of MNPs and the way they behave in vivo. 


\section{References}

1. Wu W, Wu Z, Yu T, Jiang C, Kim WS (2015) Recent progress on magnetic iron oxide nanoparticles: synthesis, surface functional strategies and biomedical applications. Sci Technol Adv Mater 16(2): 023501.

2. Saha S, Bhunia AK (2013) Synthesis of $\mathrm{Fe}_{2} \mathrm{O}_{3}$ Nanoparticles and Study of its Structural, Optical Properties. Journal of Physical Sciences 17: 191-195.

3. Akbarzadeh A, Samiei M, Davaran S (2012) Magnetic nanoparticles: preparation, physical properties, and applications in biomedicine. Nanoscale Res Lett 7(1): 144.

4. Reiss G, Hütten A (2005) Magnetic nanoparticles: Applications beyond data storage, Nature Materials 4(10): 725-726.

5. Liao W, Chen X, Chen Y, Pu S, Xia Y, et al. (2005) Tunable optical fiber filters with magnetic fluids. Appl Phys Lett 87: 151122.

6. Estelrich J, Jesús M, Martín S, Busquets MA (2015) Nanoparticles in magnetic resonance imaging: from simple to dual contrast agents. Int J Nanomedicine 10: 1727-1741.

7. Nayek C, Manna K, Bhattacharjee G, Murugavel P, Obaidat I (2017) Investigating Size- and Temperature-Dependent Coercivity and Saturation Magnetization in PEG Coated $\mathrm{Fe}_{3} \mathrm{O}_{4}$ Nanoparticles. Magnetochemistry 3(19): 1-15.

8. Arruebo M, Fernández PR, Ibarra R, Santamaría J (2007) Magnetic nanoparticles for drug delivery. Nano Today 2(3): 22-32.

9. Shin J, Anisur RM, Ko MK, Im GH, Lee JH, et al. (2009) Hollow Manganese Oxide Nanoparticles as Multifunctional Agents for Magnetic Resonance Imaging and Drug Delivery. Angew Chem Int Edit 48(2): 321-324.

10. Sharifi I, Shokrollahi H, Amiri S (2012) Ferrite-based magnetic nanofluids used in hyperthermia applications. J Magn Magn Mater 324(6): 903-915.

11. Kumar CS, Mohammad F (2011) Magnetic nanomaterials for hyperthermia-based therapy and controlled drug delivery. Adv Drug Delivery Rev 63(9): 789-808.

12. Tartaj P, Morales M, Veintemillas VS, Gonzalez CT, Serna CJ (2003) The preparation of magnetic nanoparticles for applications in biomedicine. J Phys D 36(13): 182-197.

13. Thiesen B, Jordan A (2008) Clinical applications of magnetic nanoparticles for hyperthermia. Int J Hyperthermia 24(6): 467-474.

14. Pankhurst QA, Connolly J, Jones SK, Dobson J (2003) Applications of magnetic nanoparticles in biomedicine. J Phys D Appl Phys, 36(13): 167-181.

15. Bain MSC, Yiu HH, Dobson J (2008) Magnetic nanoparticles for gene and drug delivery. Int J Nanomedicine 3(2): 169-180.

16. Laurent S, Forge D, Port M, Roch A, Robic C, et al. (2008) Magnetic Iron Oxide Nanoparticles: Synthesis, Stabilization, Vectorization, Physicochemical Characterizations, and Biological Applications. Chem Rev 108(6): 2064-2064.

17. Liu Z, Lammers T, Ehling J, Fokong S, Bornemann J, et al. Iron Oxide Nanoparticle-Containing Microbubble Composites as Contrast Agents for MR and Ultrasound Dual-Modality Imaging. Biomaterials 32(26) 6155-6163.

18. Hola K, Markova Z, Zoppellaro G, Tucek J, Zboril R (2015) Tailored functionalization of iron oxide nanoparticles for MRI, drug delivery, magnetic separation and immobilization of biosubstances. Biotechnol Adv 33(6): 1162-1176.

19. Cano M, Sbargoud K, Allard E, Larpent C (2012) Magnetic separation of fatty acids with iron oxide nanoparticles and application to extractive deacidification of vegetable oils. Green Chem 14:1786-1795.

20. Banis G, Kouli ME, Ferraro A, Molino A, Karatza D, et al. (2017) An Innovative Application of Super-Paramagnetic Iron Oxide
Nanoparticles for Magnetic Separation. Chemical Engineering Transactions 60: 85-90.

21. Koh I, Josephson L (2009) Magnetic Nanoparticle Sensors. Sensors 9(10): 8130-8145.

22. Zhang L, FX Gu, Chan JM, Wang AZ, Langer RS, et al. (2008) Nanoparticles in Medicine: Therapeutic Applications and Developments. Clinical Pharmacology \& Therapeutics 83(5): 761-769.

23. Azizian G, Alam NR, Haghgoo S, Moghimi HR, Zohdiaghdam R, et al. (2012) Synthesis route and three different core-shell impacts on magnetic characterization of gadolinium oxide-based nanoparticles as new contrast agents for molecular magnetic resonance imaging. Nanoscale Res Lett 7: 549.

24. Shen Z, Wu A, Chen X (2017) Iron Oxide Nanoparticle Based Contrast Agents for Magnetic Resonance Imaging. Mol Pharmaceutics 14(5): 1352-1364.

25. Cho SJ, Jarrett BR, Louie AY, Kauzlarich AM (2016) Goldcoated iron nanoparticles: a novel magnetic resonance agent for $T_{1}$ and $T_{2}$ weighted imaging. Nanotechnology 17(3): 640 .

26. Ma X, Gong A, Chen B, Zheng J, Chen T, et al. (2011) Exploring a new SPION-based MRI contrast agent with excellent water-dispersibility, high specificity to cancer cells and strong MR imaging efficacy. Colloids Surf B Biointerfaces 126: 44-49.

27. Borges M, Yu S, Laromaine A, Roig A, Suarez-Garcia SS, et al. (2015) Dual T1/T2 MRI contrast agent based on hybrid SPION@coordination polymer nanoparticles. RSC Adv 5: 86779-86783.

28. Shan L (2009) Superparamagnetic iron oxide nanoparticles (SPION) stabilized by alginate.

29. Madrid SIU, Pal U, Kang ZS, Kim J, Kwon H, et al. (2015) Fabrication of $\mathrm{Fe}_{3} \mathrm{O}_{4} @ \mathrm{mSiO}_{2}$ Core-Shell Composite Nanoparticles for Drug Delivery Applications. Nanoscale Res Lett 10: 217.

30. Tran N, Webster TJ (2010) Magnetic nanoparticles: biomedical applications and challenges. J Mater Chem 20(40): 8760-8767.

31. Yang HW, Hua MY, Liu HL, Yin C, Chen H, et al. (2012) Potential of magnetic nanoparticles for targeted drug delivery. Nanotechnol Sci Appl 5: 73-86.

32. Patitsa M, Karathanou K, Kanaki Z, Tzioga L, Pippa N, et al. (2017) Magnetic nanoparticles coated with polyarabic acid demonstrate enhanced drug delivery and imaging properties for cancer theranostic applications. Scientific Reports 7(775): 1-8.

33. Lv F, Fu L, Giannelis EP, Qi G (2014) Preparation of $\gamma$ - $\mathrm{Fe}_{2} \mathrm{O}_{3} / \mathrm{SiO}_{2}$-capsule composites capable of using as drug delivery and magnetic targeting system from hydrophobic iron acetylacetonate and hydrophilic $\mathrm{SiO}_{2}$ capsule. Solid State Sciences 34: 49-55.

34. Caetano BL, Guibert C, Fini R, Fresnais J, Pulcinelli SH (2016) Magnetic hyperthermia-induced drug release from ureasil-PEO- $\boldsymbol{\gamma}$ $\mathrm{Fe}_{2} \mathrm{O}_{3}$ nanocomposites. RSC Adv 6: 63291-63295.

35. Quan Q Xie J, Gao H, Yang M, Zhang F, et al. (2011) HSA Coated Iron Oxide Nanoparticles as Drug Delivery Vehicles for Cancer Therapy. Mol Pharmaceutics 8(5): 1669-1676.

36. Chertok B, Moffat BA, David AE, Yu F, Bergemann C, et al. (2008) Iron oxide nanoparticles as a drug delivery vehicle for MRI monitored magnetic targeting of brain tumors. Biomaterials 29(4): 487-496.

37. López MB, Rivas J (2013) Magnetic nanoparticle-based hyperthermia for cancer treatment, Reports of Practical Oncology \& Radiotherapy 18(6): 397-400.

38. Li W, Liu Y, Qian Z, Yang Y (2017) Evaluation of Tumor Treatment of Magnetic Nanoparticles Driven by Extremely Low Frequency Magnetic Field. Scientific Reports 7(46287). 
This work is licensed under Creative Commons Attribution 4.0 License

DOI: 10.19080/CTBEB.2018.16.555934
Your next submission with Juniper Publishers will reach you the below assets

- Quality Editorial service

- Swift Peer Review

- Reprints availability

- E-prints Service

- Manuscript Podcast for convenient understanding

- Global attainment for your research

- Manuscript accessibility in different formats ( Pdf, E-pub, Full Text, Audio)

- Unceasing customer service

Track the below URL for one-step submission https://juniperpublishers.com/online-submission.php 